\title{
LMI-based robust tracking of a class of MIMO nonlinear systems
}

\author{
ARUNIMA MUKHERJEE*D and APARAJITA SENGUPTA \\ Department of Electrical Engineering, Indian Institute of Engineering Sciences and Technology, Shibpur, \\ Howrah 711103, India \\ e-mail: amukherjee966@gmail.com
}

MS received 26 August 2018; accepted 14 July 2019

\begin{abstract}
Reference tracking problem for MIMO Lipschitz nonlinear systems is examined here. Presently a vast literature exists on observer design of unforced systems containing Lipschitz nonlinearities. However, these existing results cannot be readily extended for controller design containing reference tracking ability. Here a Linear State Variable Feedback (LSVF) controller is designed for MIMO Lipschitz nonlinear systems with norm-bounded parametric uncertainties using the concept of input to state stability Lyapunov functions. The whole problem is cast into a framework of Linear Matrix Inequalities, to exploit its numerical capabilities. Analytical proofs are supplemented with simulation examples, which show certain advantages over existing results. Apart from state feedback, observer-based output feedback is also considered for controller design.
\end{abstract}

Keywords. Nonlinear systems; Linear Matrix Inequality; reference tracking; MIMO systems; observer-based feedback controller; norm-bounded parametric uncertainties.

\section{Introduction}

Tracking has always been a challenging problem in the control system scenario, be it for linear or nonlinear systems. For nonlinear ones, the situation worsens up. When an unforced linear system is Globally Asymptotically Stable (GAS), it automatically has bounded input to bounded state (or output) properties too ('A' matrix needs to be Hurwitz) [1, 2]. The latter attribute, also denoted by $\mathcal{L}^{\infty} \rightarrow \mathcal{L}^{\infty}$ boundedness, often fails to hold for forced nonlinear systems even if they are zero-input GAS. Hence, there is a need to formulate control laws that can provide reference tracking capabilities to nonlinear systems, without affecting their stability.

Most of the existing literature on the tracking of nonlinear systems is based either on adaptive techniques [3] or on soft-computing-based control techniques [4, 5]. Adaptive controllers, in spite of being conceptually tempting, are not the obvious choice in industries yet, as they do not guarantee stability of the closed loop system (plant and adaptive controller) and often yield unsatisfactory transient behaviour. Soft-computing-based controllers, on the other hand, totally rely on the input-output mapping of the system (neglecting altogether any knowledge of the system dynamics). They thus need a long learning time before use and also have lesser flexibility. Apart from these, other techniques used are based on Dynamic Surface Control [6], Sliding Mode Control [7] and Feedback Linearization [8].

*For correspondence

Published online: 19 August 2019
All of these have complex design methodologies and mostly yield nonlinear control laws (complex to implement). Also, most of these techniques are easy to design for SISO systems [3, 5] and often pose difficulties for MIMO systems.

A major breakthrough in control engineering came with the advent of solutions of Linear Matrix Inequalities (LMIs) in the late 1990s. This method directly tests the optimization problem for its feasibility and then computes the solution through numerically efficient techniques. A large number of control problems have been formulated in the form of LMIs [9-12] and are solved using them. However, there exist few papers that employ LMIs [13-15] for tracking of nonlinear systems. Most of them contain solutions that are partly computed using LMIs and not the complete design solution. Therefore the numerical advantages are not fully exploited in the existing literature.

The paradigm of Lyapunov stability for the unforced states $x\left(t, x_{0}\right),\left(x_{0}\right.$ being the initial states) has to be shifted to accommodate bounded input bounded state stability, or namely, input state stability (ISS). Interconnected systems [16-18] and time delay system [19-23] have already been stabilized on the basis of ISS, but similar applications are not found in the field of tracking of Lipschitz nonlinear systems. The proposed LMI-based method checks for the existence of an ISS Lyapunov function for the nonlinear system. If this exists, then a controller is obtained as a solution to a set of LMIs. Thus the resultant closed loop system is always ISS. As per authors' knowledge, no such attempts have been made to date. 
The solution to each control problem is supported with analytical derivations rather than heuristic logic, thus imparting more reliability to the design. Here two approaches, i.e., one considering state feedback and another an observer-based output feedback, are used. In short, a vast class of nonlinear systems can be controlled using the method proposed in this paper.

In this paper, $\|\cdot\|$ represents the Euclidean norm. $\mathbb{R}^{+}$ represents the set of positive real numbers. $\mathbb{R}^{n \times n}$ represents the set of matrices of real numbers and of $n \times n$ dimensions. The paper is organized as follows. Section 2 lists some mathematical preliminaries required for the subsequent derivations. The problem statement is given in section 3. Section 4 contains the main results, where analytical proofs are provided for the closed loop system to be ISS and also to track the reference input. This section has two subsections 4.1 and 4.2, which, respectively, provide state feedback and observer-based feedback controller synthesis. Section 5 contains simulation results and comparative remarks that establish the superiority of the present work compared with the previously existing works. Finally, section 6 concludes the work.

\section{Preliminaries}

Definition 1 [2] If a function $\phi(x, t)$ is continuous in $t$, and if there exists a strictly positive constant $\rho$ such that

$$
\left\|\phi\left(t, x_{1}\right)-\phi\left(t, x_{2}\right)\right\| \leqslant \rho\left\|x_{1}-x_{2}\right\|
$$

$\forall x_{1}$ and $x_{2}$ in a finite neighbourhood of the origin and $\forall t$ in the interval $[t o, t o+T]$ (with $T$ being a strictly positive constant), then $\phi(x, t)$ is called a Lipschitz function, and condition (1) is known as Lipschitz condition, with constant $\rho$ as the Lipschitz constant.

Definition 2 [24] A continuous function $\alpha:[0, a) \rightarrow \mathbb{R}^{+}$ is said to be in the class $\mathcal{K}$ if

- $\alpha(0)=0$,

- it is strictly increasing.

$\alpha$ is said to be in the class $\mathcal{K}_{\infty}$ if, in addition, $\alpha: \mathbb{R}^{+} \rightarrow \mathbb{R}^{+}$ and $\alpha(r) \rightarrow \infty$ as $r \rightarrow \infty$.

Definition 3 [24] A continuous function $\beta:[0, a) \rightarrow \mathbb{R}^{+}$ is said to be in the class $\mathcal{K} \mathcal{L}$ if

- for specific $t, \beta(b, t)$ belongs to class $\mathcal{K}$ w.r.t. $b$,

- for specific $b, \beta(b, t)$ is decreasing w.r.t. $t$,

- $\beta(b, t) \rightarrow 0$ as $t \rightarrow \infty$.

For further reading, see section 2.9 [25].

Definition 4 [24] Consider the system $\dot{x}=f(x, r)$ where $f: D \times D_{r} \rightarrow \mathbb{R}^{n}$ is locally Lipschitz in $x$ and $r$ and the sets $D$ and $D_{r}$ are defined by $\left.D=\left\{x \in \mathbb{R}^{n}:\|x\|<k_{1}\right)\right\}$ and
$\left.D_{r}=\left\{r \in \mathbb{R}^{m}: \sup _{(t>0)}\|r(t)\|=\left\|r_{T}(t)\right\|_{\mathcal{L}_{\infty}}<k_{2}\right)\right\} . \quad$ This system is said to be locally input state stable (ISS) if there exists a class $\mathcal{K} \mathcal{L}$ function $\beta$, class $\mathcal{K}$ function $\sigma$ and constants $k_{1}, k_{2} \in \mathbb{R}^{+}$such that

$$
\|x(t)\| \leq \beta\left(\left\|x_{0}\right\|, t\right)+\sigma\left(\left\|r_{T}(\cdot)\right\|_{\mathcal{L}_{\infty}}\right)
$$

for all $t>0,0<T<t$ and for all $x_{o} \in D$ and $r \in D_{r}$, where $r_{T}(\cdot)$ is a truncated form of $r(t)$. It is said to be globally ISS if $D=\mathbb{R}^{n}, D_{r}=\mathbb{R}^{m}$ and (2) is satisfied for any initial state $x_{0}$ and any bounded input $\mathrm{r}(\mathrm{t})$.

For brevity $r(t)$ is represented as $r$ henceforth.

Definition $5 \quad[24,25]$ A continuous function $V: D \rightarrow \mathbb{R}$ is an ISS Lyapunov function on $D$ for the system $\dot{x}=f(x, r)$ if there exist class $\mathcal{K}$ functions $\alpha_{1}, \alpha_{2}, \alpha_{3}$ and $\sigma$ such that the following two conditions are satisfied:

- $\alpha_{1}(\|x\|) \leq V(x, t) \leq \alpha_{2}(\|x\|) \forall x \in D, t>0$,

- $\dot{V}(x) \leq-\alpha_{3}(\|x\|)+\sigma(\|r\|) \forall x \in D, r \in D_{r}$.

$\mathrm{V}$ is an ISS Lyapunov function if $D=\mathbb{R}^{n}, D_{r}=\mathbb{R}^{m}$ and $\alpha_{1}$, $\alpha_{2}, \alpha_{3}$ and $\sigma \in$ class $\mathcal{K}_{\infty}$.

Lemma 1 [26] For given matrices $M$ and $N$ with appropriate dimensions and a norm-bounded time varying matrix $F(t)$, such that $\|F(t)\| \leq 1$, we have the following conditions:

- $M F(t) N+M^{T} F(t)^{T} N^{T} \leq \varsigma M M^{T}+\varsigma^{-1} N^{T} N$

- $M N+N^{T} M^{T} \leq \epsilon M^{T} J M+\epsilon^{-1} N^{T} J^{-1} N$

where $\varsigma$ and $\epsilon$ are positive scalars and $J$ is a positive definite matrix.

\section{Problem formulation}

Consider a dynamic system of the following form:

$$
\begin{aligned}
& \dot{x}(t)=f(x(t))+B_{p} u(t) \\
& y(t)=C_{p} x(t)
\end{aligned}
$$

where $x(\cdot) \in \mathbb{R}^{n}, u(\cdot) \in \mathbb{R}^{p}$ and $y(\cdot) \in \mathbb{R}^{q}$. Furthermore, $f(x)$ is continuously differentiable w.r.t. $x$ and can be converted into

$$
f(x)=A_{p} x+\phi(x)
$$

where, without loss of generality (Chapter $4,[24]), \phi(x)$ is a function obeying (1) and,

$$
\phi(0)=0
$$

with a Lipschitz constant $\rho$.

The objective is to seek a controller such that the outputs $y_{1}(t), y_{2}(t), \ldots, y_{q}(t)$ track the reference inputs $r_{1}(t), r_{2}(t)$, $\ldots, r_{q}(t)$, respectively, and ISS of the closed loop system is ensured. To incorporate tracking capabilities in the 
controller, integral state vector $X_{n}$ is included with the system dynamics. Let $e(t)$ be the tracking error defined as

$$
e(t)=r(t)-y(t)
$$

where $e(t), r(t), y(t) \in \mathbb{R}^{q}$. Dynamics of extra state $X_{n} \in \mathbb{R}^{q}$ is given by

$$
\dot{X}_{n}=e(t)
$$

Combining (3) and (7), the final closed loop system is given by

$$
\left[\begin{array}{c}
\dot{x} \\
\dot{X_{n}}
\end{array}\right]=\left[\begin{array}{cc}
A_{p} & 0 \\
-C_{p} & 0
\end{array}\right]\left[\begin{array}{c}
x \\
X_{n}
\end{array}\right]+\left[\begin{array}{c}
B_{p} \\
0
\end{array}\right] \hat{u}+\left[\begin{array}{c}
0 \\
r(t)
\end{array}\right]+\left[\begin{array}{c}
\phi(x) \\
0
\end{array}\right]
$$

where control signal $\hat{u}$ is given by

$$
\begin{aligned}
\hat{u} & =\left[\begin{array}{ll}
\bar{K} & K_{n}
\end{array}\right]\left[\begin{array}{c}
x \\
X_{n}
\end{array}\right] \\
& =K_{a} z
\end{aligned}
$$

where $\bar{K} \in \mathbb{R}^{p \times n}, K_{n} \in \mathbb{R}^{p \times q}$ and $K_{a} \in \mathbb{R}^{p \times(n+q)}$.

Equation (8) can be written in a compact form as

$$
\dot{z}=\left(A_{a}+B_{a} K_{a}\right) z+R(t)+\phi(z)
$$

where $\phi(z)$ obeys

$$
\|\phi(z)\| \leq \gamma\|z\|
$$

with $\gamma=\sqrt{\rho^{2}+0}$ (thus $\gamma=\rho$, according to [27]), and $\gamma$ is the Lipschitz constant of $\phi(z)$.

The notations have the following meanings:

$$
\begin{cases}z=\left[\begin{array}{c}
x \\
X_{n}
\end{array}\right], \quad A_{a}=\left[\begin{array}{cc}
A_{p} & 0 \\
-C_{p} & 0
\end{array}\right], & \\
B_{a}=\left[\begin{array}{c}
B_{p} \\
0
\end{array}\right], & R(t)=\left[\begin{array}{c}
0 \\
r(t)
\end{array}\right], \quad \phi(z)=\left[\begin{array}{c}
\phi(x) \\
0
\end{array}\right]\end{cases}
$$

with $z \in \mathbb{R}^{(n+q)}, A_{a} \in \mathbb{R}^{(n+q) \times(n+q)}, B_{a} \in \mathbb{R}^{(n+q) \times p}, \phi(z) \in$ $\mathbb{R}^{(n+q)}$ and $R(t) \in \mathbb{R}^{(n+q)}$.

Hence the objective is to ensure $\mathcal{L}^{\infty} \rightarrow \mathcal{L}^{\infty}$ stability of (10), with the control signal $\hat{u}$ (as in (9)) and the tracking of reference input vector $r(t)$ by the output vector $y(t)$. It may be noted that in this situation the states of this forced system do not go to the origin.

\section{Main result}

This section contains the main contribution of this paper. Here, for the forced nonlinear system (10), it is proved that when certain LMI conditions are fulfilled, there exists an ISS Lyapunov function for the system. Hence a controller is obtained such that the closed loop system has ISS and outputs track respective reference inputs. Section 4.1 contains the design for the state feedback controller, which can directly be used where all states are measurable, such as in coupled tank systems [28], etc. Section 4.2 extends this concept for systems where some states are not directly available and thus an observer is required.

\subsection{State feedback controller}

Theorem 1 provides an LMI-based design procedure to obtain controller $K_{a}$ (in (9)) such that for (10), there exists an ISS Lyapunov function. Theorem 2 verifies the fact that the stabilizing controller $K_{a}$, found by Theorem 1 , also offers tracking abilities to the closed loop system. Theorem 3 yields a controller for a system having parametric uncertainties.

4.1a Nominal state feedback controller:

Theorem 1 For system (10), there exists an ISS Lyapunov function if there exists a feasible solution to the LMI given by

$$
\left[\begin{array}{ccc}
(1,1) & I & \rho L \\
I & -\epsilon^{-1} I & 0 \\
\rho L & 0 & -\epsilon I
\end{array}\right] \preceq 0
$$

where $(1,1)=\left(A_{a} L+B_{a} H\right)+\left(A_{a} L+B_{a} H\right)^{T}+\Delta$. Then the proposed controller is given by $K_{a}=H L^{-1}$. L and $\Delta$ are unknown positive definite matrices, $H$ is an unknown matrix and $\epsilon$ is a known scalar.

Proof 1 Consider the system in (10)-(12). Let $V(z)=$ $z^{T} P z$ be a Lyapunov function, $P$ being a positive definite matrix. As $V(z)$ is positive definite, there always exists $[1,24] \mathcal{K}_{\infty}$ functions $\alpha_{1}, \alpha_{2}$ such that

$$
\alpha_{1}(\|z\|) \leq V(z) \leq \alpha_{2}(\|z\|) .
$$

Now, differentiating $V(z)$ along (10) gives

$$
\frac{\partial V(z)}{\partial t}=z^{T} \Xi z+2 z^{T} P \phi(z)+2 z^{T} P R(t)
$$

where $\quad \Xi=\left(A_{a}+B_{a} K_{a}\right)^{T} P+P\left(A_{a}+B_{a} K_{a}\right)$. Using Lemma 1, the following inequalities can be obtained:

$$
\left\{\begin{array}{c}
2 z^{T} P \phi(z) \preceq \epsilon^{-1} \phi(z)^{T} \phi(z)+\epsilon z^{T} P P z \\
2 z^{T} P R(t) \preceq R^{T} P J^{-1} P R(t)+z^{T} J z
\end{array}\right.
$$

Using (16) and (11), (15) yields

$$
\frac{\partial V(z)}{\partial t} \leq z^{T}\left\{\Xi+\epsilon P P+\epsilon^{-1} \rho^{2} I+J\right\} z+R^{T}(t) M R(t)
$$


where $M=P J^{-1} P$, a positive definite matrix.

Assume

$$
\left\{\Xi+\epsilon P P+\epsilon^{-1} \rho^{2} I+J\right\} \preceq-S
$$

where $S$ is a positive definite matrix. Applying (18) in (17), the following condition is achieved:

$$
\dot{V} \leq-z^{T} S z+R^{T}(t) M R(t) .
$$

Thus (18) $\Rightarrow(19)$. Since $S$ is a positive definite matrix and $M$ is a positive scalar, (19) can be expressed using (Lemma 3.1 [24]) class $\mathcal{K}_{\infty}$ functions $\alpha_{3}$ and $\sigma$ as

$$
\dot{V} \leq-\alpha_{3}(\|z\|)+\sigma(\|R(t)\|) .
$$

Condition (18) together with some simple manipulations and application of Schur Complement Lemma [29, 30] yields (13), with newly defined variables $L:=P^{-1}, H:=$ $K_{a} P^{-1}$ and $\Delta:=P^{-1}(J+S) P^{-1}$. Since (13) implies (14) and (20), according to Definition 5, $V(z)$ is an ISS Lyapunov function for the system (10). This in turn implies that (10) is ISS.

Remark $1 \epsilon$ used in (16) is a tuning parameter. Here $\epsilon$ and $\epsilon^{-1}$ are to be chosen according to whether $\phi(z)$ or $P z$ term (in (16)) has to be given priority over the other. Also since both $\epsilon$ and $\epsilon^{-1}$ appear in the LMI, none of them can increase or decrease independently. Therefore, for any $\epsilon$, $\epsilon^{-1}$ is not greater or less than a factor of 4 . Here $\epsilon=1$ may be the initial value to start with. Subsequently it can be tuned for getting better responses, but only within the range $0.5<\epsilon<2$.

The following subsection establishes the tracking capabilities of the closed loop system with controller $K_{a}$ (as in (9)).

\section{1b Reference tracking:}

Theorem 2 If the system (10) in closed loop with the controller $K_{a}$ (in (9)) is ISS, the tracking error (in (6)) tends to zero asymptotically.

Proof 2 For an ISS system, according to Definition 4, each state follows (2). Under the action of the controller $K_{a}$ (obtained from Theorem 1), system (10) is ISS and hence $X_{n}$ is bounded for every $\mathcal{L}_{\infty}$-bounded reference input signal $R(t)$ and any finite initial condition $x_{0}$. Thus

$$
\dot{X}_{n} \rightarrow 0
$$

asymptotically and from (7)

$$
\dot{X}_{n}=-C_{p} x+r .
$$

Hence, tracking is achieved asymptotically for every $\mathcal{L}_{\infty^{-}}$ bounded reference input signal $R(t)$ by (10) with a controller designed using Theorem 1. 4.1c Robust state feedback controller: Since all practical systems contain uncertainty, in this section, systems with norm-bound parametric uncertainties [26] are considered. The system matrices are now $\hat{A}_{p}$ and $\hat{B}_{p}$, such that

$$
\begin{aligned}
& \hat{A}_{p}=A_{p}+M F(t) N_{a}, \\
& \hat{B}_{p}=B_{p}+M F(t) N_{b} .
\end{aligned}
$$

Here $F(t)$ is a norm-bound parametric uncertainty, such that $\|F(t)\| \leq 1 . M, N_{a} \in \mathbb{R}^{n \times n}$ and $N_{b} \in \mathbb{R}^{n}$ are known matrices that contain the information on how $F(t)$ affects the system matrices. For analysis of such a system, the original system (8) is taken, with $A_{p}$ and $B_{p}$ replaced by $\hat{A}_{p}$ and $\hat{B}_{p}$, respectively. Hence, (8) is transformed to a compact form similar to (10) as

$$
\dot{z}=\hat{A}_{a} z+\hat{B}_{a} \hat{u}_{r}+R(t)+\phi(z)
$$

where the symbols have the following meaning:

$$
\left\{\begin{array}{l}
\hat{A}_{a}=A_{a}+\tilde{M} \tilde{F}(t) \tilde{N}_{a} \\
\hat{B}_{a}=B_{a}+\tilde{M} \tilde{F}(t) \tilde{N}_{b} \\
\tilde{M}=\left[\begin{array}{cc}
M & 0 \\
0 & 0
\end{array}\right], \quad \tilde{F}(t)=\left[\begin{array}{cc}
F & 0 \\
0 & 0
\end{array}\right] \\
\tilde{N}_{a}=\left[\begin{array}{cc}
N_{a} & 0 \\
0 & 0
\end{array}\right], \quad \tilde{N}_{b}=\left[\begin{array}{c}
N_{b} \\
0
\end{array}\right], \quad \hat{u}_{r}=\hat{K}_{a} z
\end{array}\right.
$$

and $A_{a}, B_{a}$ are the same as in (12). The following theorem yields a controller that ensures the existence of an ISS Lyapunov function for the forced uncertain system in (23).

Theorem 3 For the nonlinear system of the form (23), there exists an ISS Lyapunov function if there exists a feasible solution to the LMI given by

$$
\left[\begin{array}{ccccc}
(1,1) & I & L \tilde{N}_{a}^{T} & H^{T} \tilde{N}_{b}^{T} & \rho L \\
I & -\epsilon^{-1} I & 0 & 0 & 0 \\
\tilde{N}_{a} L & 0 & -\varepsilon_{1} I & 0 & 0 \\
\tilde{N}_{b} H & 0 & 0 & -\varepsilon_{2} I & 0 \\
\rho L & 0 & 0 & 0 & -\epsilon I
\end{array}\right] \preceq 0
$$

where $(1,1)=\left(A_{a} L+B_{a} H\right)+\left(A_{a} L+B_{a} H\right)^{T}+\Delta+\left(\varepsilon_{1}+\right.$ $\left.\varepsilon_{2}\right) \tilde{M} \tilde{M}^{T}$. Here the controller is given by $\hat{K}_{a}=H L^{-1} . L, \Delta$ are unknown positive definite matrices, $H$ is an unknown matrix, $\varepsilon_{1}, \varepsilon_{2}$ are unknown positive scalars and $\epsilon$ is a known scalar (and can be obtained as in Remark 1).

Proof 3 Proceeding along the same lines as in Theorem 1, a condition (similar to (18)) is obtained, given by

$$
\Pi+\Delta+\epsilon I+\epsilon^{-1} \rho^{2} L L \preceq 0
$$

where $\Pi=\hat{A}_{a} L+L \hat{A}_{a}^{T}+\hat{B}_{a} H+H^{T} \hat{B}_{a}^{T}, \Delta=L J L, L=P^{-1}$ and $H=\hat{K}_{a} P^{-1}$. 
Expanding $\hat{A}_{a}$ and $\hat{B}_{a}$ and then using Lemma 1 , the following is obtained:

$$
\Upsilon+\Delta+\epsilon I+\epsilon^{-1} \rho^{2} L L+\Upsilon_{1}+\Upsilon_{2}+\Upsilon_{3} \preceq 0
$$

where

$$
\left\{\begin{array}{l}
\Upsilon=\left(A_{a} L+B_{a} H\right)+\left(A_{a} L+B_{a} H\right)^{T}, \\
\Upsilon_{1}=\varepsilon_{1}^{-1} L \tilde{N}_{a}^{T} \tilde{N}_{a} L, \\
\Upsilon_{2}=\varepsilon_{2}^{-1} H^{T} \tilde{N}_{b}^{T} \tilde{N}_{b} H \\
\Upsilon_{3}=\left(\varepsilon_{1}+\varepsilon_{2}\right) \tilde{M} \tilde{M}^{T} .
\end{array}\right.
$$

Using the Schur Complement Lemma on (27), the LMI given in Theorem 3 is obtained. With similar arguments as in Theorem 1, it can be shown that (23) is ISS.

\subsection{Observer-based output feedback controller}

In section 4.1, all states of the system are assumed to be measurable, but there are systems, where all states are not always physically available. For such cases, an observer is required, for obtaining the states and thus to implement the feedback control law. In the subsequent discussion, the design of an observerbased output feedback controller is carried out for (3).

4.2a Observer design: A reformulation of Theorem 1 in [12] is given in the form of Lemma 2, which will be used in the observer-based controller design, described later in the section.

Lemma 2 For a system described in (3) if there exists a positive definite matrix $Y$, a matrix $Z$ and a positive scalar $\delta$ such that (29) is feasible, then with a gain matrix $G=$ $Y^{-1} Z$, there exists an observer such that the estimation error $\eta$ converges to zero exponentially:

$$
\left[\begin{array}{cc}
\Omega+\delta \rho^{2} I & Y \\
\star & -\delta I
\end{array}\right] \prec 0
$$

where $\Omega=A_{p}^{T} Y+Y A_{p}-C_{p}^{T} Z^{T}-Z C_{p}$.

Proof 4 The observer to be designed is given by

$$
\dot{\hat{x}}=A_{p} \hat{x}+B_{p} u+\phi(\hat{x})+G C_{p}(x-\hat{x})
$$

with observer gain $\mathrm{G}$, estimated state $\hat{x}$ and estimation error $\eta=x-\hat{x}$. The dynamics of the estimation error is governed by

$$
\dot{\eta}=\left(A_{p}-G C_{p}\right) \eta+\phi(x)-\phi(\hat{x}) .
$$

Let

$$
V_{n}=\eta^{T} Y \eta
$$

be the Lyapunov function, with $Y$ being a positive definite matrix. Differentiating $V_{n}$ along (31), the following is obtained:

$$
\dot{V}_{n}=\eta^{T} \Theta \eta+2\{\phi(x)-\phi(\hat{x})\}^{T} \eta
$$

where $\Theta=\left[\left(A_{p}-G C_{p}\right)^{T} Y+Y\left(A_{p}-G C_{p}\right)\right]$.

Using (5) and Lemma 1, (33) can be written as

$$
\dot{V}_{n} \leq \eta^{T}\left[\Theta+\delta \rho^{2} I+\delta^{-1} Y Y\right] \eta .
$$

Suppose $\left[\Theta+\delta \rho^{2} I+\delta^{-1} Y Y\right]=U$. Here, $\eta$ converges to zero if

$$
\dot{V}_{n} \leq \eta^{T} U \eta<0
$$

i.e., if

$$
\Theta+\delta \rho^{2} I+\delta^{-1} Y Y \prec 0 .
$$

Applying Schur complement on (36) and defining the variables $Z:=Y G$ we get the LMI described in Lemma 2.

Now, using (32) and (35) and the fact that $U$ is a negative definite matrix, the following is obtained:

$$
\dot{V}_{n}<f_{1} V_{n}
$$

where $f_{1}=\frac{\lambda_{\max } U}{\lambda_{\min } Y}$ and it is a negative constant. Thus, the error dynamics in (31) converges to zero exponentially [2].

4.2b Observer-based controller design: For this design, the states used by the feedback controller (in 9) are the estimated state vector $\hat{x}$ (as in (30)). The system state vector $x$ is replaced by its estimated state vector $\hat{x}$ in (9). Thus, (8) is transformed into the following:

$$
\left[\begin{array}{c}
\dot{x} \\
\dot{X_{n}}
\end{array}\right]=\left[\begin{array}{cc}
A_{p} & 0 \\
-C_{p} & 0
\end{array}\right]\left[\begin{array}{c}
x \\
X_{n}
\end{array}\right]+\left[\begin{array}{c}
\phi(x) \\
0
\end{array}\right]+\left[\begin{array}{c}
B_{p} \\
0
\end{array}\right] \hat{u}_{e}+R(t)
$$

where

$$
\hat{u}_{e}=\left[\begin{array}{ll}
K & K_{n}
\end{array}\right]\left[\begin{array}{c}
\hat{x} \\
X_{n}
\end{array}\right] .
$$

Next, the final closed loop system, including estimation error dynamics (31) and system dynamics in (37) and (38), is given by

$$
\dot{z}_{o}=A_{o} z_{o}+\phi_{o}+B_{o} u_{o}+R_{o}(t)
$$

where the notations have the following meanings:

$$
\left\{\begin{array}{l}
z_{o}=\left[\begin{array}{l}
z \\
\eta
\end{array}\right], \quad A_{o}=\left[\begin{array}{cc}
A_{a} & 0 \\
0 & \left(A_{p}-G C_{p}\right)
\end{array}\right], \\
B_{o}=\left[\begin{array}{c}
B_{a} \\
0
\end{array}\right], \quad R_{o}(t)=\left[\begin{array}{c}
R(t) \\
0
\end{array}\right], \quad \phi_{o}=\left[\begin{array}{c}
\phi(z) \\
\phi(x)-\phi(\hat{x})
\end{array}\right], \\
u_{o}=K_{o} z_{o}, \quad K_{o}=\left[\begin{array}{lll}
K & K_{n} & -K
\end{array}\right] .
\end{array}\right.
$$


Here $z, A_{a}, B_{a}, R(t)$ and $\phi(z)$ (used in (40)) have the same meaning as in (12). $G, \eta$ are the same as in (31). $K_{o}$ is the feedback controller gain for (39). The following theorem now yields the controller $K_{o}$.

Theorem 4 For (3), if an observer gain $G$ be found from Lemma 2 and additionally if (41) is feasible, then there exists a controller gain $K_{o}=\mathrm{r}^{-1}$ for the observer-based closed loop system (39). In that case there exists an ISS Lyapunov function for (39), it is ISS and reference tracking is also achieved:

$$
\left[\begin{array}{ccc}
\Xi & I & \Gamma Q \\
I & -\epsilon^{-1} I & 0 \\
\Gamma Q & 0 & -\epsilon I
\end{array}\right] \prec 0
$$

where $\Xi=Q A_{o}^{T}+A_{o} Q+\Upsilon^{T} B_{o}^{T}+B_{o} \Upsilon+\Theta$. Here $Q$ and $\Theta$ are unknown positive definite matrices, $\Upsilon$ is an unknown matrix and $\epsilon$ is a known scalar.

Proof 5 Let $V_{o}=z_{o}^{T} W z_{o}$ be a Lyapunov function, with $W$ being a positive definite matrix. Differentiating $V_{o}$ along (39) gives

$$
\dot{V}_{o}=z_{o}^{T} \Sigma z_{o}+2 z_{o}^{T} W \phi_{o}+2 z_{o}^{T} W R_{o}(t)
$$

with $\Sigma=A_{o}^{T} W+W A_{o}+K_{o}^{T} B_{o}^{T} W+W B_{o} K_{o}$. Here, using (5) and (11)

$$
\phi_{o}^{T} \phi_{o} \preceq \Gamma^{2} z_{o}^{T} z_{o}
$$

with $\Gamma^{2}=\gamma^{2}+\rho^{2}$. Using (43) and Lemma 1, the following is obtained:

$$
\dot{V}_{o} \leq-z_{o}^{T} T z_{o}+R_{o}^{T} N R_{o}
$$

where $T=-\left\{\Sigma+\epsilon^{-1} \Gamma^{2} I+\epsilon W W+J\right\}$ and $N=W J^{-1} W$.

In a similar way as in Theorem 1 , for $V_{o}$ to be an ISS Lyapunov function for (39), $T$ and $N$ should be positive definite. This is true only if the following condition is true:

$$
\Sigma+\epsilon^{-1} \Gamma^{2} I+\epsilon W W+J \preceq 0
$$

Using some simple manipulations and the Schur complement Lemma, and defining new variables $Q:=W^{-1}, \Upsilon:=$ $K_{o} W^{-1}$ and $\Theta:=W^{-1} J W^{-1}$, (45) yields the LMI in (41). Hence if (41) is obeyed, then there exists a $V_{o}$ that is an ISS Lyapunov function for (39). Thus (39) is ISS.

In the same line as done before (in Theorem 2), it can be shown that (39) will also track reference inputs efficiently.

4.2c Robust observer-based controller design: With a similar approach as in section 4.1c, system matrices $A_{p}$ and $B_{p}$ are replaced with their uncertain counterparts $\hat{A}_{p}$ and $\hat{B}_{p}$ in section $4.2 \mathrm{~b}$. Hence, (39) is transformed to a compact form, given by

$$
\dot{z}_{o}=\hat{A}_{o} z_{o}+\phi_{o}+\hat{B}_{o} \hat{u}_{o}+R_{o}(t)
$$

where the notations have the following meanings:

$$
\begin{gathered}
\hat{A}_{o}=A_{o}+\tilde{M}_{o} \tilde{F}(t) \tilde{N}_{a o}, \\
\hat{B}_{o}=B_{o}+\tilde{M}_{b o} \tilde{F}(t) \tilde{N}_{b o}, \\
\tilde{M}_{o}=\left[\begin{array}{ccc}
M & 0 & 0 \\
0 & 0 & 0 \\
0 & 0 & M
\end{array}\right], \quad \tilde{M}_{b o}=\left[\begin{array}{ccc}
M & 0 & 0 \\
0 & 0 & 0 \\
0 & 0 & 0
\end{array}\right], \\
\tilde{F}(t)=\left[\begin{array}{ccc}
F & 0 & 0 \\
0 & 0 & 0 \\
0 & 0 & 0
\end{array}\right], \quad \tilde{N}_{a o}=\left[\begin{array}{ccc}
N_{a} & 0 & 0 \\
0 & 0 & 0 \\
0 & 0 & N_{a}
\end{array}\right], \\
\tilde{N}_{b o}=\left[\begin{array}{c}
N_{b} \\
0 \\
0
\end{array}\right], \quad, \\
\hat{u}_{o}=\hat{K}_{o} z_{o},
\end{gathered}
$$

and $A_{o}, B_{o}, z_{o}, \phi_{o}$ and $R_{o}(t)$ are the same as in (40).

Theorem 5 For (3), if an observer gain $G$ be found from Lemma 2 and additionally if (52) is feasible, then there exists a controller gain $\hat{K}_{o}=G_{o} L_{o}^{-1}$ for the observer-based closed loop system (46). In that case (46) is ISS, and reference tracking is also achieved:

$$
\left[\begin{array}{ccccc}
(1,1) & I & L_{o} \tilde{N}_{a o}^{T} & G_{o}^{T} \tilde{N}_{b o}^{T} & \Gamma L_{o} \\
I & -\epsilon^{-1} I & 0 & 0 & 0 \\
\tilde{N}_{a o} L_{o} & 0 & -\varepsilon_{1} I & 0 & 0 \\
\tilde{N}_{b o} G_{o} & 0 & 0 & -\varepsilon_{2} I & 0 \\
\Gamma L_{o} & 0 & 0 & 0 & -\epsilon I
\end{array}\right] \preceq 0
$$

where $(1,1)=\left(A_{o} L_{o}+B_{o} G_{o}\right)+\left(A_{o} L_{o}+B_{o} G_{o}\right)^{T}+\Delta_{o}+$ $\varepsilon_{1} \tilde{M}_{o} \tilde{M}_{o}^{T}+\varepsilon_{2} \tilde{M}_{b o} \tilde{M}_{b o}^{T} . L_{o}, \Delta_{o}$ are unknown positive definite matrices, $G_{o}$ is an unknown matrix, $\varepsilon_{1}, \varepsilon_{2}$ are unknown positive scalars and $\epsilon$ is a known scalar.

Proof 6 Proceeding along the same lines as in Theorem 4, a condition (similar to (45)) is obtained and given by

$$
\bar{\Sigma}+\epsilon^{-1} \Gamma^{2} L_{o} L_{o}+\epsilon I+\Delta_{o} \preceq 0
$$

with $\bar{\Sigma}=L_{o} \hat{A}_{o}^{T}+\hat{A}_{o} L_{o}+G_{o}^{T} \hat{B}_{o}^{T}+\hat{B}_{o} G_{o}, \Delta_{o}=L_{o} J L_{o}, L_{o}=$ $W^{-1}$ and $G_{o}=\hat{K}_{o} W^{-1}$. Expanding $\hat{A}_{o}$ and $\hat{B}_{o}$ and applying Lemma 1, (53) gives (54):

$$
\Lambda+\epsilon I+\epsilon^{-1} \Gamma^{2} L_{o} L_{o}+\chi_{1}+\chi_{2} .
$$

Here $\Lambda=L_{o} A_{o}^{T}+A_{o} L_{o}+G_{o}^{T} B_{o}^{T}+B_{o} G_{o}+\chi_{3}$,

$$
\begin{aligned}
& \chi_{1}=\varepsilon_{1}^{-1}\left(\tilde{N}_{a o} L_{o}\right)^{T}\left(\tilde{N}_{a o} L_{o}\right), \\
& \chi_{2}=\varepsilon_{2}^{-1}\left(\tilde{N}_{b o} L_{o}\right)^{T}\left(\tilde{N}_{b o} L_{o}\right) \text { and }
\end{aligned}
$$




$$
\chi_{3}=\varepsilon_{1}^{-1} \tilde{M}_{o} \tilde{M}_{o}^{T}+\varepsilon_{2}^{-1} \tilde{M}_{b o} \tilde{M}_{b o}^{T}+\Delta_{o} .
$$

Application of Schur complement Lemma (54) yields (52). With similar arguments as in Theorem 4 it can be shown that there exists an ISS Lyapunov function for (46) and it is ISS, together with reference tracking abilities.

Remark 2 Theorems 1, 3, 4 and 5 can easily be used for SISO systems; therefore, no separate proofs are given for the same.

\section{Simulation results and comparisons}

This section illustrates the applicability of the proposed method on practical systems and also highlights the major advantages of the proposed approach over the existing ones.

Remark 3 The control signals $\hat{u}, \hat{u}_{r}, u_{o}$ and $\hat{u}_{o}$ obtained using Theorems 1,3,4, and 5, respectively, are linear combinations of states, rather than their nonlinear functions as in $[5-7,31]$ and many more, which are difficult to implement.

The first example is of a SISO system containing a Lipschitz nonlinearity and norm-bounded parametric uncertainty. It is also assumed that all the states are available for measurement; thus, a state feedback controller is designed.

Example 1 The applicability of Theorem 3 is shown by the following mass-spring system example (inspired by [1]), since a mass-spring-damper is the elementary part of many mechanical systems. Consider a mechanical system consisting of a mass-spring, where mass $M_{a}$ moves on a horizontal surface and is also tied to a vertical surface as shown in figure 1 through a nonlinear hard spring. This mass is under the influence of an external force $T$. The displacement from the reference position is represented by $y . F_{f}$ is a frictional force and $F_{s p}$ is the restorative force due to the nonlinear hard spring. These forces are related to each other with the following equations:

$$
\begin{aligned}
M_{a} \ddot{y}+F_{f}+F_{s p} & =T \\
F_{f} & =c \dot{y} \\
F_{s p} & =K\left(y+a^{2} y^{3}\right)
\end{aligned}
$$

where $\frac{K}{M_{a}}=1, \frac{c}{M_{a}}=1$ and $\frac{K a^{2}}{M_{a}}=0.6$.

The system matrices as in (23) are obtained, by choosing the states $y$ and $\dot{y}$ as $x_{1}$ and $x_{2}$, respectively, and are given by $A_{p}=\left[\begin{array}{cc}0 & 1 \\ -1 & -1\end{array}\right], \quad B_{p}=\left[\begin{array}{l}0 \\ 1\end{array}\right], \quad C_{p}=\left[\begin{array}{ll}1 & 0\end{array}\right], \quad M=$ $\left[\begin{array}{cc}0.1 & 0.05 \\ -2 & 0.1\end{array}\right], N_{a}=\left[\begin{array}{cc}0.1 & 0 \\ 0 & 0.1\end{array}\right]$ and $\phi(x)=\left[\begin{array}{c}0 \\ 0.6\left(x_{1}\right)^{3}\end{array}\right]$.

Using Theorem 3, with $\epsilon=2$, the resulting controller is given as $\hat{K}_{a}=10^{3} \times\left[\begin{array}{lll}-1.2382 & -0.0702 & 1.1203\end{array}\right]$. For a

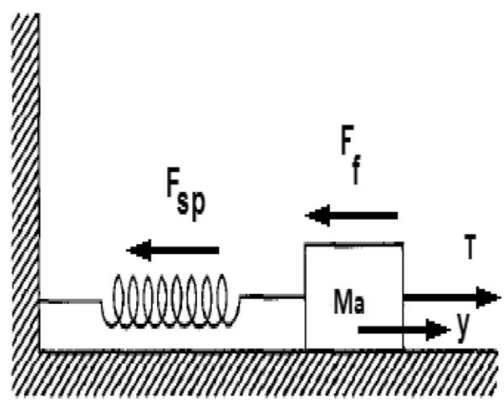

Figure 1. Mass-spring mechanical system.

reference input of a square wave (amplitude 3 and time period of $62.83 \mathrm{~s}$ ) and norm-bounded uncertainty $\tilde{F}(t)$, the evolution of the output w.r.t. time can be seen in figure 2 .

The output here is state $x_{1}$ and it is seen to track the reference input.

The second example is of a MIMO Lipschitz nonlinearity, containing norm-bound parametric uncertainty. Here also it is assumed that all the states are available for sensing; thus, a state feedback controller is designed.

Example 2 For illustrating the use of Theorem 3 for MIMO system, this numerical example is considered. For a system described in (23), the nominal system matrices $\left(A_{p}\right.$, $B_{p}$ and $\left.C_{p}\right)$ and constant matrices $\left(M, N_{a}\right.$ and $\left.N_{b}\right)$, which map how uncertainties affect the system dynamics, are given by $A_{p}=\left[\begin{array}{lll}1 & 0 & 0 \\ 0 & 2 & 1 \\ 0 & 1 & 3\end{array}\right], \quad B_{p}=\left[\begin{array}{ll}1 & 0 \\ 0 & 1 \\ 0 & 0\end{array}\right]$, $C_{p}=\left[\begin{array}{lll}1 & 0 & 0 \\ 0 & 0 & 2\end{array}\right], \quad M=(0.1) I_{3 \times 3}, \quad N_{a}=I_{3 \times 3}$, $N_{b}=\left[\begin{array}{cc}0.1 & 0.2 \\ 0.2 & 0 \\ 0 & 0.3\end{array}\right], \phi(z)=\left[\begin{array}{c}0 \\ 0.3 \sin \left(x_{1}\right) \\ 0\end{array}\right]$ and the reference $r(t)=\left[\begin{array}{l}0.1 \\ 0.2\end{array}\right]$.

Using Theorem 3 with $\epsilon=0.5, \hat{K}_{a}$ is obtained as $\hat{K}_{a}=\left[\begin{array}{ccccc}-10.97 & 4.231 & 43.159 & 15.29 & -9.18 \\ -3.358 & -59.83 & -565.92 & 4.618 & 114.54\end{array}\right]$.

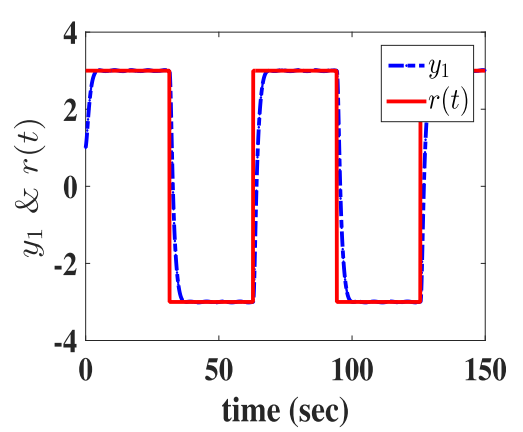

Figure 2. Evolution of output and reference input w.r.t. time. 


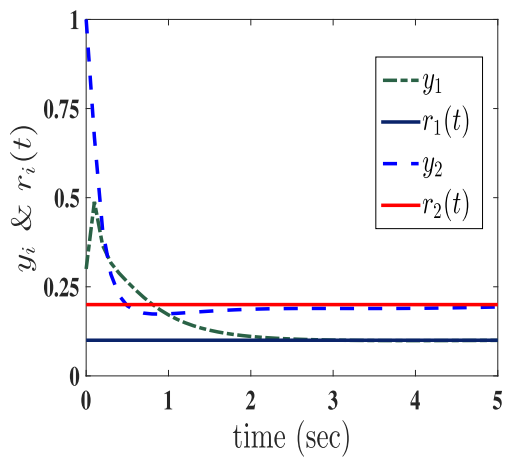

Figure 3. Reference signal tracking.

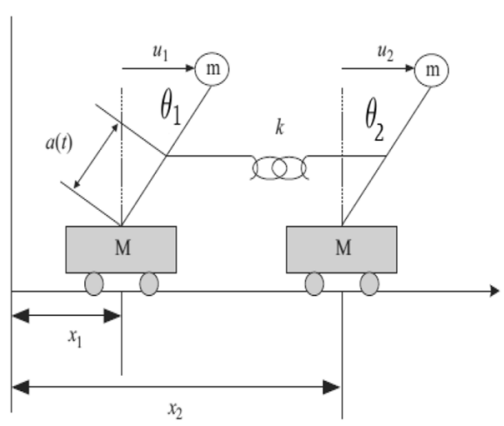

Figure 4. Inverted pendulum system.

The outputs $y_{1}$ and $y_{2}$ in figure 3 are seen to satisfactorily track the reference inputs $r_{1}(t)$ and $r_{2}(t)$, respectively.

Remark 4 Here $B_{p}$ is a full column matrix, which is more common in practice rather than $B_{p}$ being full rank as required in [31] . Also the condition $B_{p} B_{p r}=I$ [31] is not required in our method, where $B_{p r}$ is the pseudo-inverse of $B_{p}$.

The third example considers a MIMO system that contains Lipschitz nonlinearities and norm-bounded parametric uncertainty. In this case, the states are not measurable and thus state estimation is required. Therefore, in this example, an observer-based output feedback control is implemented.

Example 3 Consider two inverted pendulums that are connected by a moving spring mounted on two carts [32]. Their configuration is shown in figure 4. It is assumed that the pivot position of the moving spring is a function of time and it can change with the length $l$ of the pendulums. The motion of the carts is specified to be sinusoidal trajectories. The input to each pendulum is the torque $u_{i}, i=1,2$ applied at the pivot point. The dynamic equations of the inverted double pendulums can be described as

$$
\begin{aligned}
& \ddot{\theta}_{1}=\frac{g}{c l} \theta_{1}+\frac{1}{c m l^{2}} u_{1}+f_{1}-\frac{m}{M} \dot{\theta}_{1}^{2} \sin \left(\theta_{1}\right) \\
& \ddot{\theta}_{2}=\frac{g}{c l} \theta_{2}+\frac{1}{c m l^{2}} u_{2}+f_{2}-\frac{m}{M} \dot{\theta}_{2}^{2} \sin \left(\theta_{2}\right)
\end{aligned}
$$

where $f_{1}$ and $f_{2}$ are given by

$$
\begin{aligned}
& f_{1}=\left\{\frac{k[a(t)-c(t)]}{c m l^{2}}\left[-a(t) \theta_{1}+a(t) \theta_{2}-x_{1}+x_{2}\right]\right. \\
& f_{2}=\left\{\frac{k[a(t)-c(t)]}{c m l^{2}}\left[-a(t) \theta_{2}+a(t) \theta_{1}-x_{2}+x_{1}\right]\right.
\end{aligned}
$$

where $\theta_{i}$ are the angles with respect to vertical axes, $\dot{\theta}_{i}$ are the angular velocities of the pendulums, $u_{1}$ and $u_{2}$ are the control torques applied to the pendulums, $k$ is the spring constant, $g=1 \mathrm{~N}$ is the gravity constant, $a(t)$ is a function of time $t$ and $a(t) \in[0, l], c$ is a fractional constant and is equal to $m /(M+m), l=1 \mathrm{~m}, k=1 \mathrm{~N} / \mathrm{m}, M=m=10 \mathrm{~kg}$, $a(t)=\sin (\omega t), x_{1}=\sin \left(\omega_{1} t\right), x_{2}=\sin \left(\omega_{2} t\right)+L$ are the motion trajectories of two carts at time $t, L=2 \mathrm{~m}, \omega_{1}=2$, $\omega_{2}=3$ and $\omega=5$.

Defining the states as $x_{11}=\theta_{1}, x_{12}=\dot{\theta}_{1}, x_{21}=\theta_{2}$ and $x_{22}=\dot{\theta_{2}}$, the state space equivalent of (55), the system matrices, defined as in (3),(4) and (22) are found to be the following: $A_{p}=\left[\begin{array}{cccc}0 & 1 & 0 & 0 \\ 2 & 0 & 0 & 0 \\ 0 & 0 & 0 & 1 \\ 0 & 0 & 2 & 0\end{array}\right], \quad B_{p}=\left[\begin{array}{cc}0 & 0 \\ 0.2 & 0 \\ 0 & 0 \\ 0 & 0.2\end{array}\right]$, $C_{p}=\left[\begin{array}{llll}1 & 0 & 0 & 0 \\ 0 & 0 & 1 & 0\end{array}\right], M=\left[\begin{array}{cccc}0 & 0 & 0 & 0 \\ -0.1 & 0 & 0.1 & 0 \\ 0 & 0 & 0 & 0 \\ 0.1 & 0 & -0.1 & 0\end{array}\right]$, $F(t)=\sin ^{2}(5 t), \quad N_{a}=I_{4}, \quad N_{b}=0, \quad \phi(x)=$ $\left[\begin{array}{c}0 \\ -x_{12}^{2} \sin \left(x_{11}\right) \\ 0 \\ -x_{22}^{2} \sin \left(x_{21}\right)\end{array}\right]$. Initially the observer gain, $G$ is obtained using Lemma 2 and then using Theorem $5, \hat{K}_{o}$ is obtained. Thus, observer-based feedback control is applied to the MIMO Lipschitz nonlinear system.

Here $G=\left[\begin{array}{cc}4.5728 & 0 \\ 7.0709 & 0 \\ 0 & 4.5728 \\ 0 & 7.0709\end{array}\right]$,

$\hat{K}=\left[\begin{array}{cccc}-1837.9 & -210 & 12.40 & 01.40 \\ 12.40 & 01.40 & -1837.9 & -210\end{array}\right]$ and $\hat{K} n=$ $\left[\begin{array}{cc}1619.3 & -11.0 \\ -11.0 & 1619.3\end{array}\right]$ with $\epsilon=0.5$. The trajectories for $y_{1}$ and $y_{2}$ for references $r_{1}(t)=0$ and $r_{2}(t)=0$ are given in figures 5 and 6 , respectively.

Remark 5 The responses in figures 5 and 6 can be compared to figures 2 and 3 in [32], respectively. Here, two advantages can be seen in the present work:

- Better tracking is achieved, as there is no steady state error present here.

- No overshoot or undershoot is observed.

Moreover in the present work, another reference set is also considered. Figure 7 gives the tracking response of $y_{1}$ 


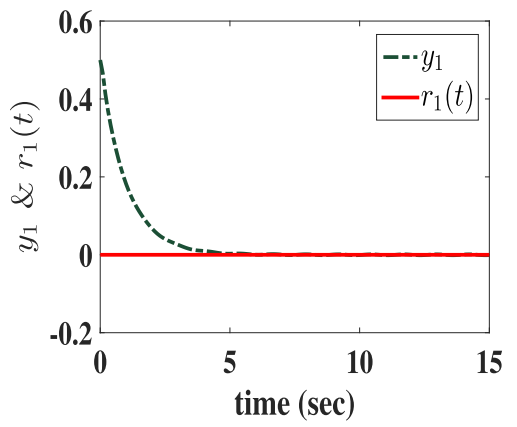

Figure 5. Reference signal tracking by $y_{1}$ for $r_{1}(t)=0$.

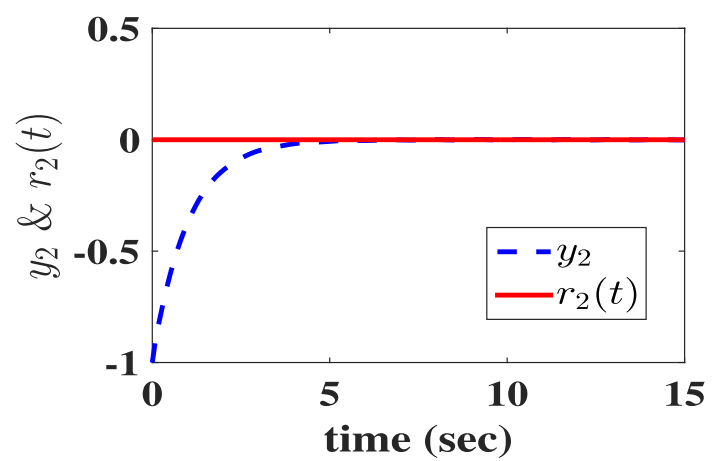

Figure 6. Reference signal tracking by $y_{2}$ for $r_{2}(t)=0$.

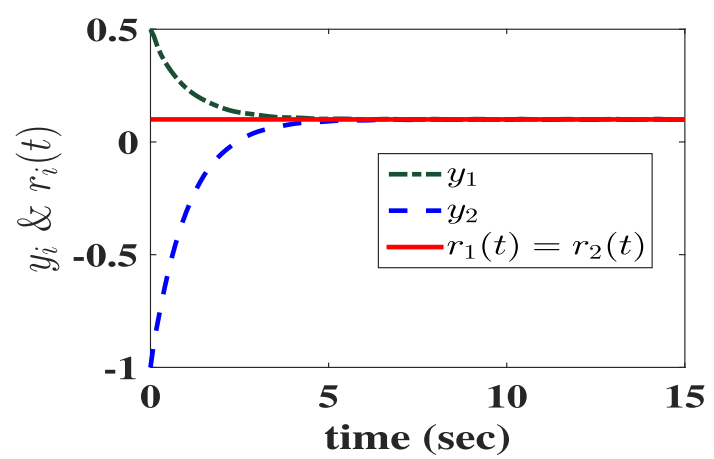

Figure 7. Reference signal tracking by $y_{1}$ and $y_{2}$.

and $y_{2}$ for reference inputs $r_{1}(t)=0.1$ and $r_{2}(t)=0.1$, respectively.

\section{Conclusion}

Here an LMI-based controller design technique is developed for MIMO Lipschitz nonlinear systems, containing norm-bounded uncertainties. The resultant closed loop system is ISS and has the capability of reference tracking. When proposed LMIs are satisfied, the controller obtained is in the form of linear (in state) control laws and hence may be easily implemented physically. For systems where states are inaccessible, an observer-based feedback controller is also proposed. The design examples demonstrate the simplicity and advantages of the proposed method.

\section{Acknowledgements}

This work is financially supported by the Indian Institute of Engineering Science and Technology, Shibpur, West Bengal, India.

\section{References}

[1] Khalil H K and Grizzle J W 1996 Nonlinear systems. Englewood Cliffs, NJ: Prentice-Hall

[2] Slotine J J E, Li W et al 1991 Applied nonlinear control. Englewood Cliffs, NJ: Prentice-Hall

[3] Sokolov V F 2003 Adaptive suboptimal tracking for a firstorder object under Lipschitz uncertainty. Automation and Remote Control 64: 457-467

[4] Zhang T and Ge S S 2009 Adaptive neural network tracking control of MIMO nonlinear systems with unknown dead zones and control directions. IEEE Transactions on Neural Networks 20: 483-497

[5] Li P and Yang G H 2008 Adaptive fuzzy control of unknown nonlinear systems with actuator failures for robust output tracking. In: Proceedings of American Control Conference, pp. $4898-4903$

[6] Swaroop D, Hedrick J K, Yip P P and Gerdes J C 2000 Dynamic surface control for a class of nonlinear systems. IEEE Transactions on Automatic Control 45: 1893-1899

[7] Slotine J J and Sastry S S 1983 Tracking control of nonlinear systems using sliding surfaces, with application to robot manipulators. International Journal of Control 38: 465-492

[8] Pop C I and Dulf E H 2011 Robust feedback linearization control for reference tracking and disturbance rejection in nonlinear systems. London: INTECH Open Access Publisher

[9] Zemouche A and Boutayeb M 2013 On LMI conditions to design observers for Lipschitz nonlinear systems. Automatica 49: $585-591$

[10] Kheloufi H, Zemouche A, Bedouhene F and Boutayeb M 2013 On LMI conditions to design observer based controllers for linear systems with parameter uncertainties. Automatica 49: $3700-3704$

[11] Ibrir S and Diopt S 2008 Novel LMI conditions for observerbased stabilization of Lipschitzian nonlinear systems and uncertain linear systems in discrete-time. Applied Mathematics and Computation 206: 579-588

[12] Phanomchoeng G and Rajamani R 2010 Observer design for Lipschitz nonlinear systems using Riccati equations. In: Proceedings of American Control Conference (ACC), pp. 6060-6065

[13] Tseng C S, Chen B S and Uang H J 2001 Fuzzy tracking control design for nonlinear dynamic systems via TS fuzzy model. IEEE Transactions on Fuzzy Systems 9: 381-392 
[14] Zhang Q, Sijun Y, Yan L and Xinmin W 2011 An enhanced LMI approach for mixed $\mathrm{H}_{2} / H \infty$ flight tracking control. Chinese Journal of Aeronautics 24: 324-328

[15] Dahmani H, Pagès O, El Hajjaji A and Daraoui N 2013 Observer-based tracking control of the vehicle lateral dynamics using four-wheel active steering. In: Proceedings of the 16th International IEEE Conference on Intelligent Transportation Systems (ITSC 2013), pp. 360-365

[16] Jiang Z P, Teel A R and Praly L 1994 Small-gain theorem for ISS systems and applications. Mathematics of Control, Signals and Systems 7: 95-120

[17] Dashkovskiy S, Rüffer B S and Wirth F R 2007 An ISS small gain theorem for general networks. Mathematics of Control, Signals, and Systems 19: 93-122

[18] Dashkovskiy S N, Rüffer B S and Wirth F R 2010 Small gain theorems for large scale systems and construction of ISS Lyapunov functions. SIAM Journal on Control and Optimization 48: 4089-4118

[19] Teel A R 1998 Connections between Razumikhin-type theorems and the ISS nonlinear small gain theorem. IEEE Transactions on Automatic Control 43: 960-964

[20] Dashkovskiy S and Naujok L 2010 Lyapunov-Razumikhin and Lyapunov-Krasovskii theorems for interconnected ISS time-delay systems. In: Proceedings of the 19th International Symposium on Mathematical Theory of Networks and Systems (MTNS10), pp. 1179-1184

[21] Fridman E, Dambrine M and Yeganefar N 2008 On input-tostate stability of systems with time-delay: a matrix inequalities approach. Automatica 44: 2364-2369

[22] Tiwari S, Wang Y and Jiang Z P 2009 A nonlinear smallgain theorem for large-scale time delay systems. In: Proceedings of the 48th IEEE Conference on Decision and Control 2009 held jointly with the 2009 28th Chinese Control Conference CDC/CCC 2009, pp. 7204-7209
[23] Aleksandrov A Y, Hu G D and Zhabko A P 2014 Delayindependent stability conditions for some classes of nonlinear systems. IEEE Transactions on Automatic Control 59: 2209-2214

[24] Marquez H J 2003 Nonlinear control systems: analysis and design. Hoboken, NJ: Wiley-Interscience

[25] Sontag E D 2008 Input to state stability: basic concepts and results. In: Nistri P and Stefani G (Eds.) Nonlinear and Optimal Control Theory, Lecture Notes in Mathematics. Berlin: Springer, pp. 163-220

[26] Liu P L 2009 Robust exponential stability for uncertain timevarying delay systems with delay dependence. Journal of the Franklin Institute 346: 958-968

[27] Eriksson K, Estep D and Johnson C 2004 Vector-valued functions of several real variables. In: Calculus in Several Dimensions, Calculus in Several Dimensions, Applied Mathematics: Body and Soul. Berlin: Springer, pp. 789814

[28] Aydin S and Tokat S 2008 Sliding mode control of a coupled tank system with a state varying sliding surface parameter. In: Proceedings of the 2008 International Workshop on Variable Structure Systems, pp. 355-360

[29] Scherer C and Weiland S 2000 Linear matrix inequalities in control. In: Lecture Notes. Delft: Dutch Institute for Systems and Control

[30] Boyd S P, El Ghaoui L, Feron E and Balakrishnan V 1994 Linear matrix inequalities in system and control theory. Philadelphia: SIAM

[31] Rehan M, Hong K S and Ge S S 2011 Stabilization and tracking control for a class of nonlinear systems. Nonlinear Analysis: Real World Applications 12: 1786-1796

[32] Liu Y J, Tong S C and Li T S 2011 Observer based adaptive fuzzy tracking control for a class of uncertain nonlinear MIMO systems. Fuzzy Sets and Systems 164: 25-44 